\title{
Lateral chest X-ray for physicians
}

\author{
Khalid A Gaber MSc MRCP ${ }^{1}$ Clive R McGavin FRCP ${ }^{2}$ Irving P Wells FRCR $^{3}$
}

J R Soc Med 2005;98:310-312

Despite advances in radiology, the chest X-ray (CXR) has survived as the most frequently requested examination. About 6.7 million are performed each year in the UK. ${ }^{1}$ The radiation dose of a frontal CXR is equivalent to only three days' exposure to natural background radiation (effective dose $0.02 \mathrm{mSv}^{2}$ The lateral $\mathrm{CXR}$ is less frequently requested and more difficult to interpret. Nevertheless it contains much information on the thoracic cage, pleura, lungs, pericardium, heart, mediastinum and upper abdomen. Many junior doctors, at ease with the frontal CXR, seem intimidated by the lateral film. Uncertain when to request or how to interpret, they may retreat expensively and unnecessarily into computerized tomography.

The lateral CXR is performed with the side of interest closer to the X-ray film, giving an effective dose of $0.05 \mathrm{mSv}$. If the side is not specified, a left lateral is usually taken. In this paper we offer a brief guide to demystify and clarify this cheap and useful investigation. Essentially there are four indications and four main abnormalities to interpret.

\section{INDICATIONS}

When a lateral film is desired, the reasons should be stated on the request form. The four indications are to localize a lesion seen on frontal CXR, to clarify lobar collapse/ consolidation, to explore a retrosternal or retrocardiac shadow or to confirm the presence of encysted fluid in the oblique fissure (pseudotumour).

\section{NORMAL LANDMARKS}

The fissures are important landmarks on a lateral CXR, becoming visible when the X-ray beam passes parallel to them. The oblique fissure begins posteriorly at T4/5 level, passing through the hilum. The left is steeper and finishes $5 \mathrm{~cm}$ behind the costophrenic angle whereas the right ends just behind the angle. The horizontal fissure runs anteriorly from the hilum separating the right upper lobe from the middle lobe. On the left there is no horizontal fissure.

'Department of Respiratory Medicine, Bristol Royal Infirmary, Bristol BS2 8HW; and Departments of ${ }^{2}$ Respiratory Medicine and ${ }^{3}$ Radiology, Derriford Hospital, Plymouth PL6 8DH, UK

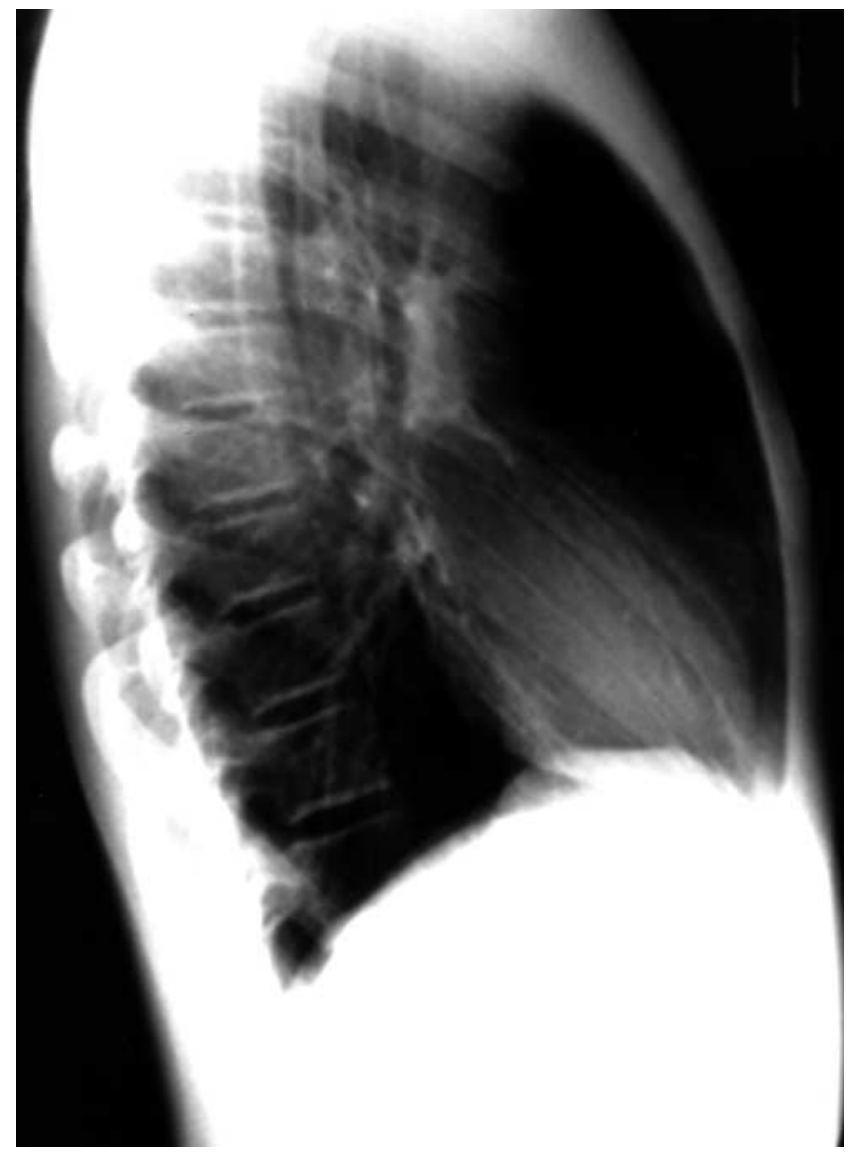

Figure 1 Normal lateral chest X-ray

Both hemidiaphragms should be visible, silhouetted by the lung air against the fluid-density of the abdominal contents. The anterior end of the left hemidiaphragm may be lost against the heart. The diaphragms can be distinguished from each other if there is clearly gastric air under one, or if one can be seen to be inserted into the magnified ribs further from the X-ray plate.

Vertebral bodies become darker as they proceed caudally (Figure 1) until they reach the diaphragm-because there is more soft tissue and less lung tissue at the apex but more lung tissue and less soft tissue at the bases.

The retrocardiac and retrosternal spaces are normally seen as dark areas about equal in size and lucency.

\section{ABNORMALITIES IN THE LANDMARKS}

The fissures will be displaced in the direction of a collapsing lobe (Figure 2). With simple consolidation there will 


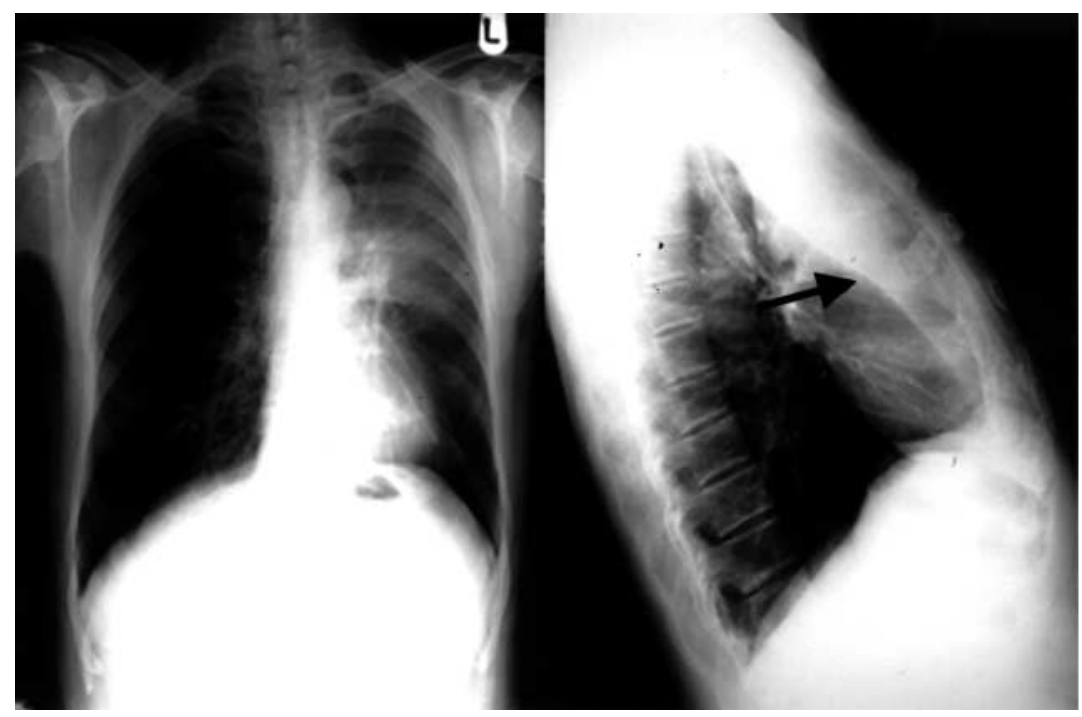

Figure 2 Left upper lobe collapse. Oblique fissure moves anteriorly (arrow)

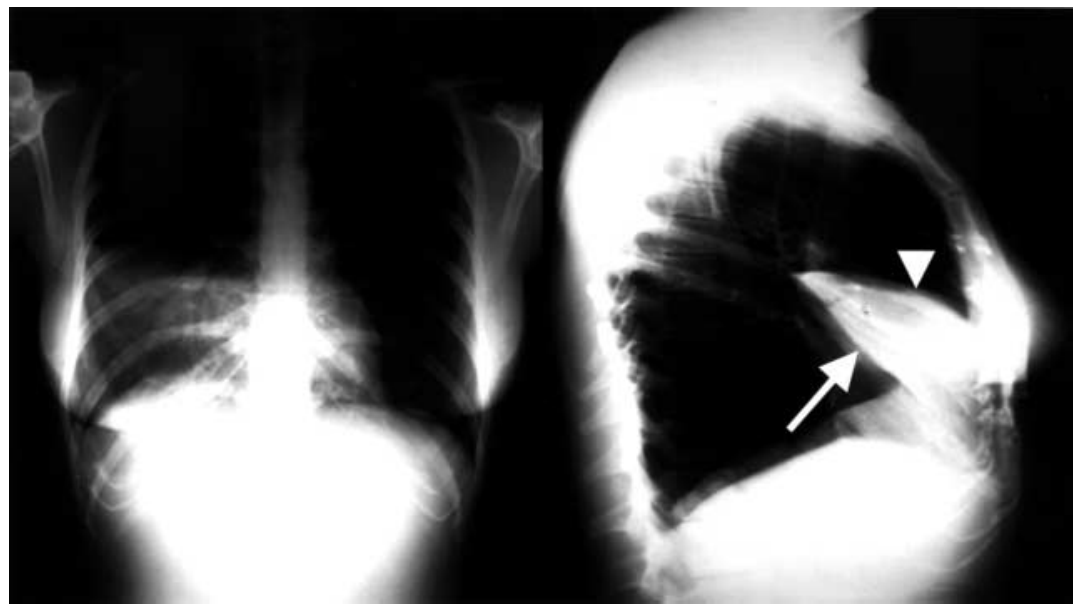

Figure 3 Middle lobe consolidation. Note oblique fissure (arrow) and transverse fissure (arrowhead)

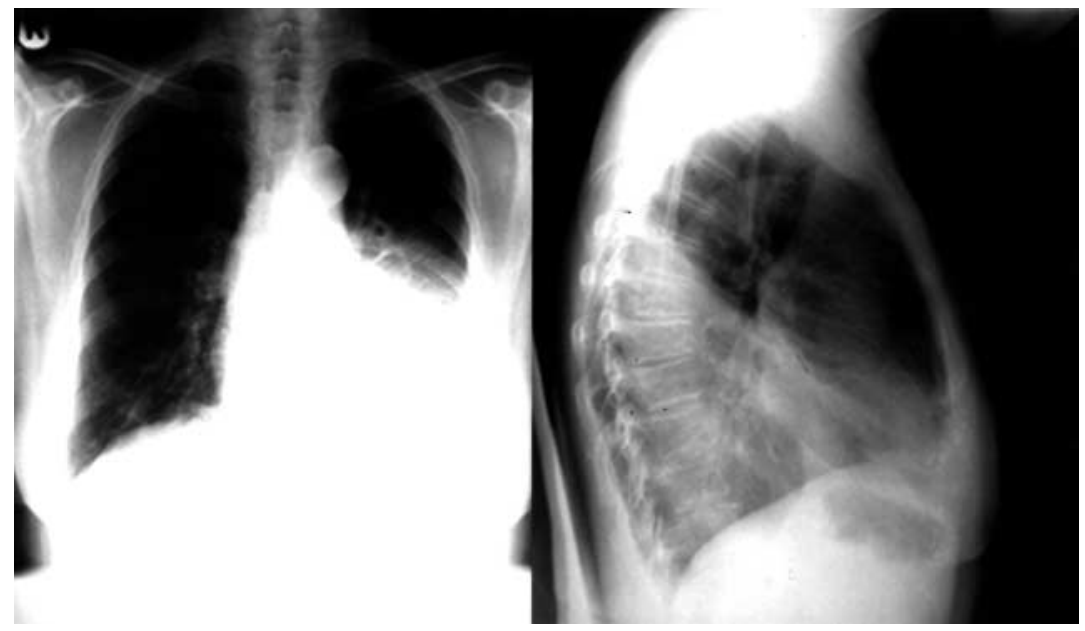

Figure 4 Left lower lobe consolidation. Note loss of the left hemidiaphragm and of caudal vertebral lucency 
be opacification up to the straight edge of a nondisplaced fissure (Figure 3); displacement of a fissure results from collapse. The bi-convex shadow of fluid in a fissure will clarify a pseudotumour seen on the frontal view.

The diaphragms: when only one diaphragm is visible the silhouette of the other must have been lost. That is to say, there is fluid density as opposed to air density above the diaphragm - usually indicating effusion or consolidation. If no other border is seen higher up, this suggests complete opacification of that hemithorax.

Vertebral bodies: if these remain white as they proceed caudally, this indicates increased radiodensity as opposed to radiolucency at the lung base, caused by pleural disease, consolidation or a mass lesion (Figure 4).
The retrosternal space is enlarged in pulmonary overinflation (emphysema) and opacified by anterior mediastinal disease such as thymoma or lymphoma. The retrocardiac space will appear opaque where there is effusion, consolidation or a mass. Other abnormalities may be evident such as pectus excavatum, pericardial calcification and hiatus hernia. The lateral CXR is seldom indicated for examining the hilum in an adult but in children it can be helpful in investigation of lymphadenopathy, for example in primary tuberculosis.

\section{REFERENCES}

1 National Radiological Protection Board. Frequency of Medical and Dental $X$-Ray Examination in the UK 1997-1998. London: NRPB, 2000

2 Royal College of Radiologists. Making the Best Use of a Department of Clinical Radiology: Guidelines for Doctors, 3rd edn. London: RCR, 1995:12 